\title{
Limits on the effective quark radius from inclusive ep scattering at HERA
}

\author{
Oleksii Turkot* on behalf of the ZEUS collaboration \\ DESY \\ E-mail: oleksii.turkot@desy.de
}

\begin{abstract}
The high precision HERA combined measurement of inclusive deep inelastic cross sections in neutral and charged current $e p$ scattering, corresponding to a luminosity of about $1 \mathrm{fb}^{-1}$, permits searches for new contributions to electron-quark scattering beyond the Standard Model up to $\mathrm{TeV}$ scales. A new approach to beyond the Standard Model analysis of the inclusive ep data is presented; simultaneous fits of parton distribution functions and contributions of "new physics" processes were performed. Results are presented considering a finite radius of quarks within the quark form-factor model. The resulting $95 \%$ C.L. upper limit on the effective quark radius is $0.43 \cdot 10^{-16} \mathrm{~cm}$.
\end{abstract}

The European Physical Society Conference on High Energy Physics

5-12 July

Venice, Italy

${ }^{*}$ Speaker. 


\section{Introduction}

Measurements of deep inelastic scattering (DIS) of electrons ${ }^{1}$ on protons at HERA provide an importand input for determination of proton structure and allow searches for "beyond the Standard Model" (BSM) contributions to electron-quark scattering at the scales higher than the centre-ofmass energy. The HERA collider operated at electron beam energy of $27.5 \mathrm{GeV}$ and proton beam energies of 920, 820, 575 and $460 \mathrm{GeV}$, which allowed the two collaborations, H1 and ZEUS, to explore a large phase space in negative four-momentum-transfer squared, $Q^{2}$, and Bjorken $x$, $x_{B j}$. In total about $1 \mathrm{fb}^{-1}$ of data were collected by the two experiments, divided almost equally between $e^{+} p$ and $e^{-} p$ scattering. All final inclusive DIS neutral current (NC) and charged current (CC) cross-section measurements from ZEUS and H1 experiments were combined [1], providing one coherent set of data.

The availability of high precision NC and CC cross sections over large phase space made HERA data an essential ingredient in the estimation of all recent high-precision PDFs and allows to evaluate precise PDFs using solely HERA data. In case of an unrecognized BSM contributions in the data the determination of PDFs within the Standard Model would result in biased PDF distributions. If cross-section predictions obtained with such PDFs would be used in the BSM analysis evaluated limits on BSM processes will be overestimated. To avoid such possible bias in the quark radius analysis the combined HERA DIS data was used in simultaneous fits of parton distribution functions and effective quark-radius parameter.

\section{The ZCIPDFs PDF set}

To predict cross sections without BSM contributions the Standard Model perturbative QCD analysis [2] was performed similarly to that for the HERAPDF2.0 determination [1]. The proton PDFs were described by 14 parameters at the starting scale of $1.9 \mathrm{GeV}^{2}$ and evolved to other scales using DGLAP equations. The PDF parameters were fitted to the combined HERA inclusive DIS cross-section values $\mu_{0}^{i}$ assuming a Gaussian distribution of statistical, uncorrelated and systematic uncertainties with the following $\chi^{2}$ formula:

$$
\chi^{2}(m, s)=\sum_{i} \frac{\left[m^{i}+\sum_{j} \gamma_{j}^{i} m^{i} s_{j}-\mu_{0}^{i}\right]^{2}}{\left(\delta_{i, \text { stat }}^{2}+\delta_{i, \text { uncor }}^{2}\right)\left(\mu_{0}^{i}\right)^{2}}+\sum_{j} s_{j}^{2} .
$$

There $\gamma_{j}^{i}, \delta_{i, \text { stat }}$ and $\delta_{i, \text { uncor }}$ are the relative correlated systematic, relative statistical and relative uncorrelated systematic uncertainties of the input data, respectively. The vector $m$ represents the set of pQCD cross-section predictions $m^{i}$ which depends on the PDF parameters $p$ at the starting scale, and the components $s_{j}$ of the vector $s$ represent the correlated systematic shifts of the cross sections in units of the relative correlated systematic uncertainties $\gamma_{j}^{i}$. The summations extend over all data points $i$ and all correlated systematic uncertainties $j$.

The resulting PDFs set is referred to as ZCIPDF and gluon, valence quarks and sea distributions of ZCIPDFs at the scale of $Q^{2}=10 \mathrm{GeV}^{2}$ are presented and compared to the distributions of HERAPDF2.0 in Fig. 1.

\footnotetext{
${ }^{1}$ Here and later, the word "electron" refers to both electrons and positrons, unless otherwise stated.
} 


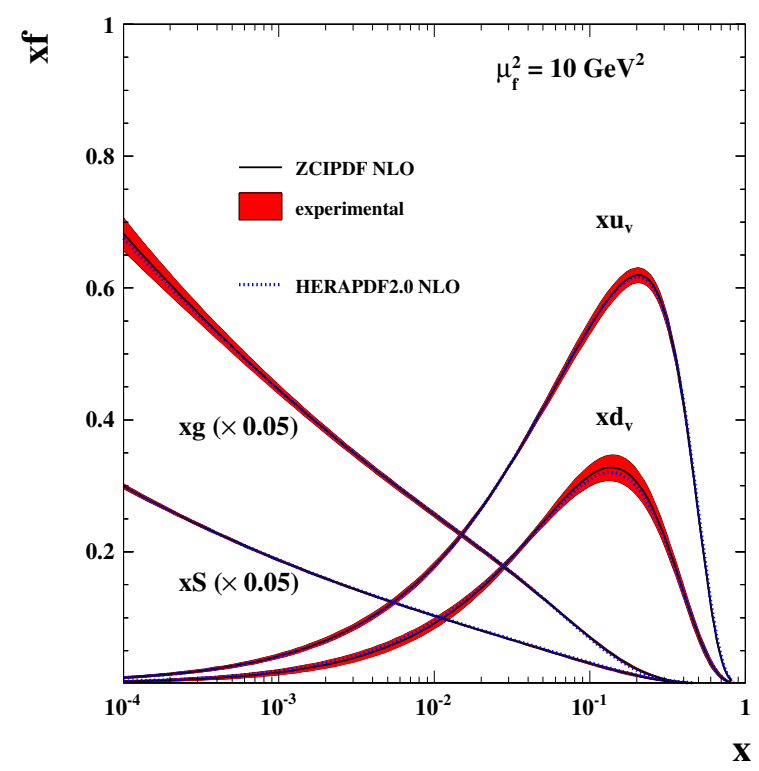

Figure 1: The PDF set ZCIPDF with experimental uncertainty at the factorisation scale $\mu_{\mathrm{f}}^{2}=Q^{2}=10 \mathrm{GeV}^{2}$. The central values of HERAPDF2.0 NLO are shown with the dashed line.

\section{Quark form-factor model}

One of the possible BSM scenarios is to assign an effective finite radius to electrons and/or quarks and, if the expected deviations are small, the predictions for such scenarios can be calculated by modifying the SM cross-section predictions with a semi-classical form-factor [3]:

$$
\frac{d \sigma}{d Q^{2}}=\frac{d \sigma^{\mathrm{SM}}}{d Q^{2}}\left(1-\frac{R_{e}^{2}}{6} Q^{2}\right)^{2}\left(1-\frac{R_{q}^{2}}{6} Q^{2}\right)^{2},
$$

where $R_{e}^{2}$ and $R_{q}^{2}$ are the mean-square radii of the electron and the quark, respectively. In the presented analysis the electron was assumed to be point-like, $R_{e}^{2} \equiv 0$, and, to maintain the generality of the approach, both positive and negative values of quark radius were considered. Neutral current and charged current DIS cross sections are modified by Eq. 3.1 in a similar way.

\section{QCD+BSM limit-setting procedure}

The quark-radius parameter $R_{\mathrm{q}}^{2}$ Fit can be estimated simultaneously with PDFs as an additional fit parameter. To check if such a fit would prevent biases in the fitted CI parameter values, a test on the generated pseudodata has been performed. The pseudodata sets were prepared in the same $\left(Q^{2}, x\right)$ grid as the data for different values of the quark radius parameter $R_{\mathrm{q}}^{2}$ True from the predictions of ZCIPDF, modified according to Eq. 3.1. The uncertainties for the resulting crosssection values were taken to be the same as for the data. The QCD fit simultaneously estimated PDF parameters and $R_{\mathrm{q}}^{2 \text { Fit }}$ on the obtained sets of pseudodata. The results are displayed in Fig. 2 (left) as 
solid blue circles. For comparison, a two-step fit was also performed on the same pseudodata sets, first estimating PDF parameters within the Standard Model and afterwards fitting $R_{\mathrm{q}}^{2}$ Fit with PDF parameters fixed to the results of the first fit. Results of the two-step fit are presented in Fig. 2 (left) with open red circles. They show a bias in the CI parameter values that is not present in the case of the combined fit.
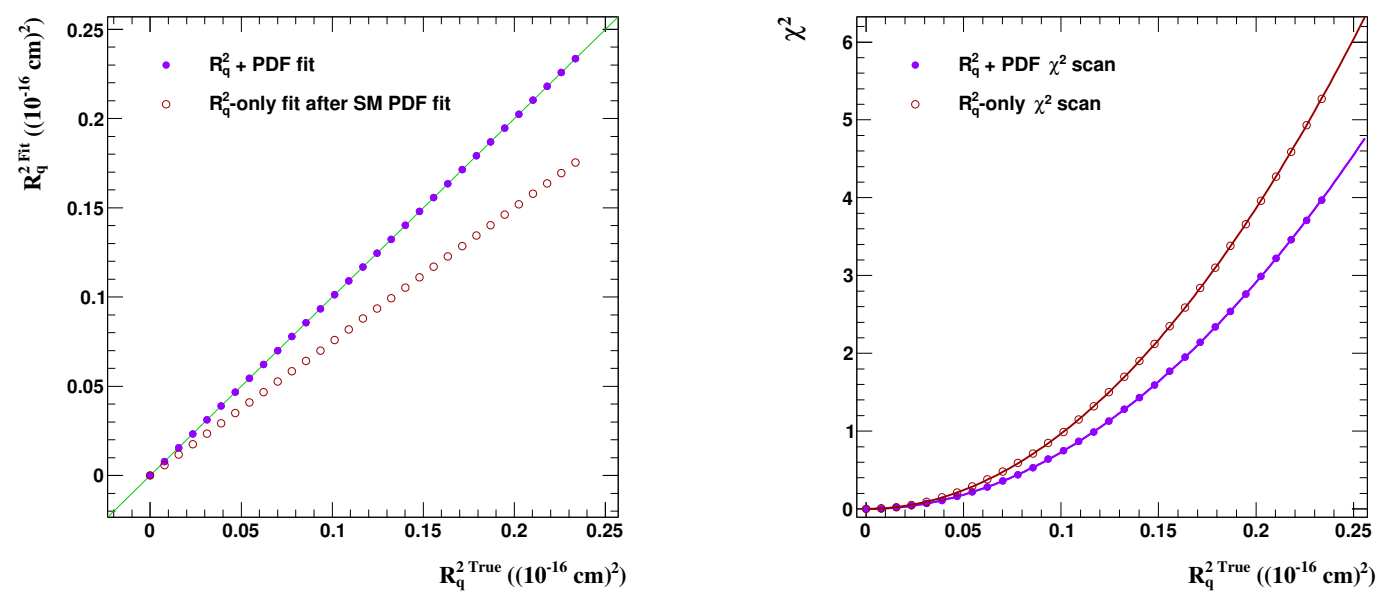

Figure 2: $R_{\mathrm{q}}^{2}$ Fit results of Monte Carlo replicas fits (left) and $\chi^{2}$ distributions (right) with the combined procedure (solid blue circles) and the procedure with fixed PDFs (open red circles). The pseudodata for the fits are obtained from the Standard Model cross-section predictions (right) and by modifying the Standard Model predictions with the quark-radius form-factor $R_{\mathrm{q}}^{2}$ True (left).

To show that the analysis procedure with fixed PDFs could result in an overestimated $R_{\mathrm{q}}$ limits, the distributions of $\chi^{2}\left(R_{\mathrm{q}}^{2}\right.$ True $)$ were calculated using the pseudodata set generated with $R_{\mathrm{q}}^{2}$ True $=0$. The results obtained with the combined procedure are shown in Fig. 2 (right) as solid blue circles and with the procedure with fixed PDFs as open red circles. The distribution for the procedure with fixed PDFs can be described with a narrower parabola and thus would result in stronger limits than the combined procedure.

The limits on the quark-radius form-factor were derived in a frequentist approach [4] based on fits to large sets of Monte Carlo replicas [2]. Monte Carlo replicas are sets of cross-section values in the same $\left(Q^{2}, x\right)$ grid as data. They were generated by randomly varying cross-section predictions from ZCIPDF scaled according to Eq. 3.1 within the statistical and systematic uncertainties taken from the data:

$$
\mu^{i}=\left[m_{0}^{i}+\sqrt{\delta_{i, \text { stat }}^{2}+\delta_{i, \text { uncor }}^{2}} \cdot \mu_{0}^{i} \cdot r_{i}\right] \cdot\left(1+\sum_{j} \gamma_{j}^{i} \cdot r_{j}\right) .
$$

There, $\mu^{i}$ is the resulting cross-section replica, $m_{0}^{i}$ is scaled ZCIPDF cross-section prediction, $\mu_{0}^{i}$, $\delta_{i, \text { stat }}^{2}, \delta_{i, \text { uncor }}^{2}$ and $\gamma_{j}^{i}$ are the same data cross section, relative statistical, uncorrelated and correlated systematic uncertainties as in Eq. 2.1, respectively, and $r_{i}$ and $r_{j}$ represent random numbers taken from a normal distribution. 
Up to 5000 sets of replicas $\mu^{i}$ were generated for each value of $R_{\mathrm{q}}^{2}$ True and used for a combined QCD fit simultaneously estimating PDF parameters and the quark-radius parameter $R_{\mathrm{q}}^{2}$ Fit. The value of quark radius fitted on data, $R_{\mathrm{q}}^{2}$ Data, was taken as a test statistic, to which the values of $R_{\mathrm{q}}^{2}$ Fit were compared and probability $P$ to obtain $R_{\mathrm{q}}^{2}$ Fit $<R_{\mathrm{q}}^{2}$ Data (for a limit in a positive direction, displayed in Fig. 3 (right)) or $R_{\mathrm{q}}^{2 \text { Fit }}>R_{\mathrm{q}}^{2 \text { Data }}$ (for a limit in the negative direction, displayed in Fig. 3 (left)) was evaluated. The quark-radius parameter values corresponding to $P=5 \%$ provided the $95 \%$ C.L. limits.
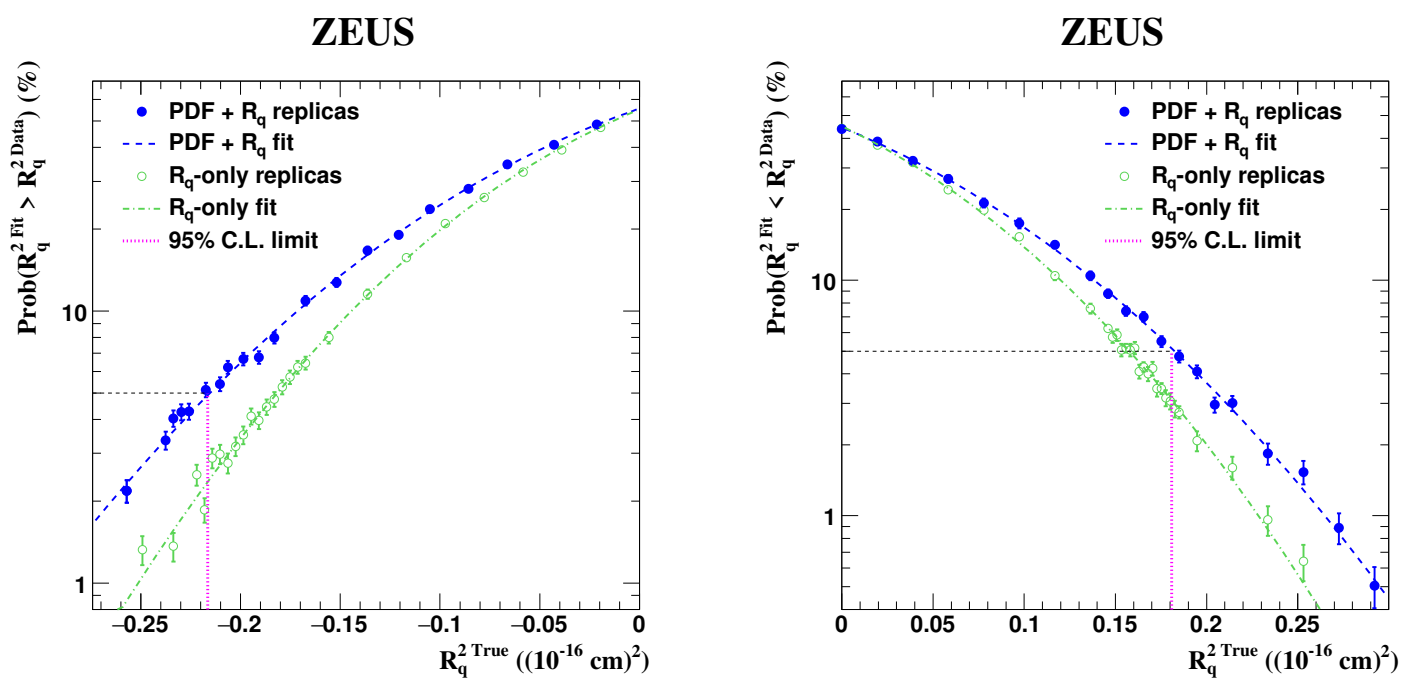

Figure 3: The probability of obtaining $R_{q}^{2 \text { Fit }}$ values greater (left) or smaller (right) than that obtained for the actual data, $R_{q}^{2 \text { Data }}$, calculated from the Monte Carlo replicas, as a function of the assumed value for the quark-radius squared, $R_{q}^{2}$ True. Points with statistical error bars represent the Monte Carlo replica sets generated for different values of $R_{q}^{2 \text { True }}$. The solid blue circles correspond to the results obtained from the simultaneous fit of $R_{q}^{2}$ and PDF parameters $\left(\mathrm{PDF}+R_{q}\right)$. For comparison, the open green circles represent the dependence obtained when fixing the PDF parameters to the ZCIPDF values $\left(R_{q}\right.$-only).

\section{Results}

The analysis of quark radius based on simultaneous fits of PDF parameters and $R_{q}^{2}$ using the sets of Monte Carlo replicas yields the 95\% C.L. limits of

$$
-\left(0.47 \cdot 10^{-16} \mathrm{~cm}\right)^{2}<R_{\mathrm{q}}^{2}<\left(0.43 \cdot 10^{-16} \mathrm{~cm}\right)^{2}
$$

Deviations of the cross sections corresponding to the 95\% C.L. exclusion limits on $R_{q}^{2}$ are compared to the combined HERA high- $Q^{2}$ NC and CC DIS data integrated over $x$ in Fig. 4 (left) and Fig. 4 (right), respectively. These limits are about $8 \%$ weaker than the limits that would be obtained for fixed PDF parameters, as demonstrated in Fig. 3. 

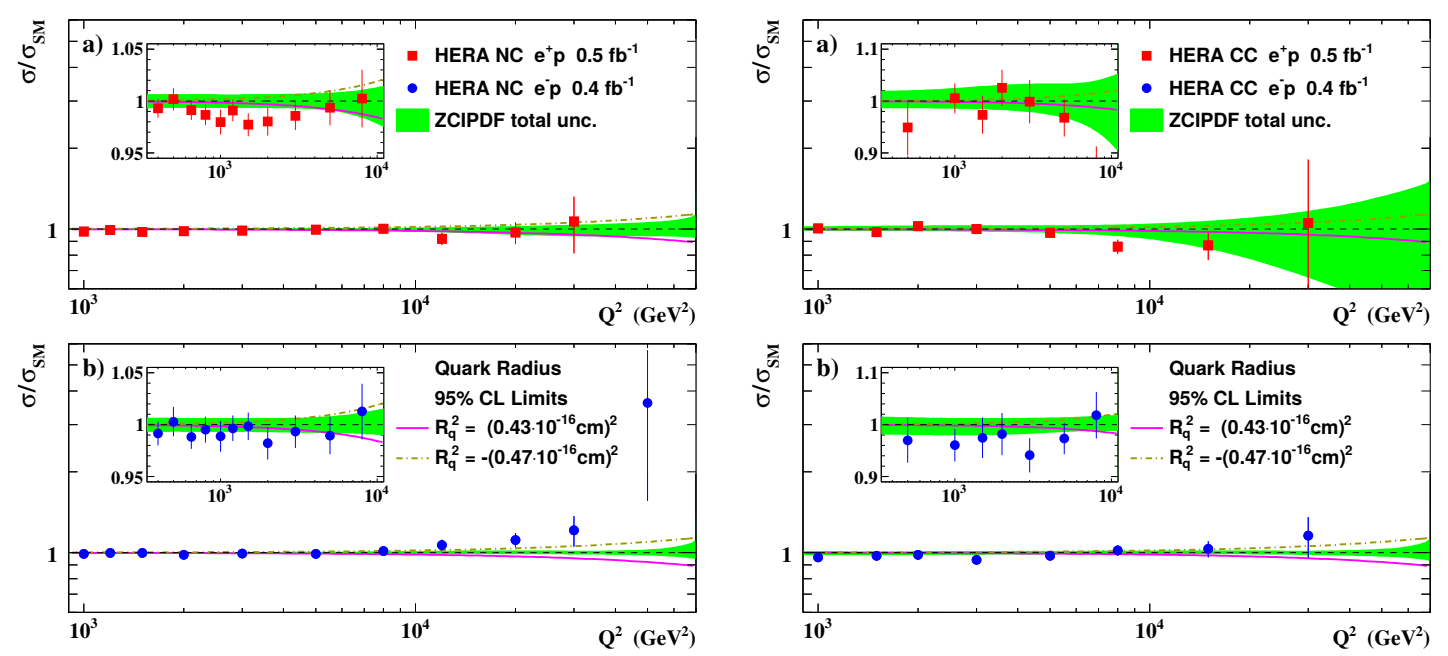

Figure 4: Combined HERA (a) $e^{+} p$ and (b) $e^{-} p$ NC (left) and CC (right) DIS data divided by the crosssection expectations from ZCIPDF and compared to the 95\% C.L. exclusion limits on the effective meansquare radius of quarks. The bands on the ZCIPDF predictions represent the total uncertainty. The insets show the comparison in the $Q^{2}<10^{4} \mathrm{GeV}^{2}$ region with a linear ordinate scale.

\section{References}

[1] H1 and ZEUS Collaborations, H. Abramowicz et al., Combination of measurements of inclusive deep inelastic $e \pm p$ scattering cross sections and QCD analysis of HERA data, Eur. Phys. J. C 75 (2015) 1 [arXiv:1506.06042]

[2] ZEUS Collaborations, H. Abramowicz et al., Limits on the effective quark radius from inclusive ep scattering at HERA, Phys. Lett. B 757 (2016) 468 [arXiv: 1604 . 01280]

[3] G. Köpp and others, Bounds on radii and magnetic dipole moments of quarks and leptons from LEP, SLC and HERA, Z. Phys. C 65 (1995) 545 [hep-ph/ 9409457$].$

[4] R. Cousins, Why isn't every physicist a Bayesian? Am. J. Phys. 63 (1995) 398. 